\title{
CARACTERIZAÇÃO DE PACIENTES COM LESÕES DE PELE HOSPITALIZADOS EM UNIDADES DE INTERNAÇÃO CLÍNICO-CIRÚRGICA
}

Rochelli Bernardes Stefanello ${ }^{1}$ https://orcid.org/0000-0002-6604-0932 Silvana Mara Janing Prazeres ${ }^{2}$ https://orcid.org/0000-0002-4071-8039 Fernanda Silva dos Santos ${ }^{2}$ Joel Rolim Mancia ${ }^{2}$ https://orcid.org/0000-0001-8613-578X https://orcid.org/0000-0001-9817-2537

Sandra Maria Cezar Leal https://orcid.org/0000-0003-4611-0988

Objetivo: caracterizar os pacientes com lesões de pele no início e no fim da internação hospitalar. Método: estudo transversal. Participaram 45 pacientes com lesões de pele. Incluidos pacientes avaliados pela enfermeira, no mínimo duas vezes durante a internação. Dados coletados do prontuário eletrônico e analisados por meio de estatística descritiva e inferencial no programa SPSS ${ }^{\circledR}$. Resultados: maioria de idosos, sexo feminino, média de idade de 67 anos, motivo de internação vascular e comorbidades associadas, tempo médio de internação,2l dias e desfecho de alta hospitalar. A ferida prevalente foi a lesão por pressão localizada na região lombo-sacra. Os ácidos graxos essenciais, seguidos do alginato de cálcio e da hidrofibra de prata, foram os mais utilizados. Os escores de classificação de risco foram Moderado/Elevado para o desenvolvimento de Lesão por Pressão. Conclusão: Conhecer as características sociodemográficas e epidemiológicas dos pacientes com lesões de pele, instrumentaliza para o planejamento e implantação da assistência integral e sistematizada.

Descritores: Ferimentos e Lesões; Bandagens; Enfermagem.

\section{CHARACTERIZATION OF PATIENTS WITH SKIN WOUNDS HOSPITALIZED ON CLINICAL/SURGICAL INPATIENT UNITS}

Objective: To characterize patients with skin lesions at the beginning and end of hospital stay. Method: cross-sectional study conducted; 45 patients with skin lesions participated. Included patients evaluated by the nurse at least twice during hospitalization. Data collected from the electronic medical record and analyzed using descriptive and inferential statistics in the SPSS® program. Results: majority of the elderly, female, mean age of 67 years, reason for vascular hospitalization and associated comorbidities, mean length of stay, 21 days and outcome of hospital discharge. The prevalent wound was the pressure injury located in the lumbosacral region. Essential fatty acids, followed by calcium alginate and silver hydrofiber, were the most used. Risk rating scores were Moderate / High for the development of Pressure Injury. Conclusion: Knowing the sociodemographic and epidemiological characteristics of patients with skin lesions, instruments for the planning and implementation of comprehensive and systematized care.

Descriptors: Wounds and Injuries; Bandages; Nursing.

\section{CARACTERIZACIÓN DE PACIENTES CON LESIONES DE LA PIEL HOSPITALIZADOS EN UNIDADES DE CIRUGÍA CLÍNICA}

Objetivo: caracterizar a los pacientes con lesiones cutáneas al inicio y al final de la estancia hospitalaria. Metodo: estudio transversal. Participaron 45 pacientes con lesiones cutáneas. Pacientes incluidos evaluados por la enfermera al menos dos veces durante la hospitalización. Datos recopilados del registro médico electrónico y analizados mediante estadísticas descriptivas e inferenciales en el programa SPSS®. Resultados: mayoría de los ancianos, mujeres, edad media de 67 años, motivo de hospitalización vascular y comorbilidades asociadas, duración media de la estancia, 21 dias y resultado del alta hospitalaria. La herida prevalente fue la lesión por presión ubicada en la región lumbosacra. Los ácidos grasos esenciales, seguidos por alginato de calcio e hidrofibra de plata, fueron los más utilizados. Los puntajes de calificación de riesgo fueron Moderado / Alto para el desarrollo de Lesión por Presión. Conclusión: Conocer las características sociodemográficas y epidemiológicas de pacientes con lesiones cutáneas, instrumentos para la planificación e implementación de atención integral y sistematizada.

Descriptores: lesiones y lesiones; Vendajes; Enfermería.

Hospital Divina Providência, Porto Alegre/RS.

${ }^{2}$ Universidade do Vale do Rio dos Sinos-UNISINOS.

Autor correspondente: Joel Rolim Mancia. - E-mail: joelrm@unisinos.br

Recebido: 10/07/2019 - Aceito: 30/12/2019 


\section{INTRODUÇÃO}

O aumento na demanda de atendimentos às pessoas com feridas passou a ser um desafio enfrentado por toda a equipe multiprofissional de saúde, especialmente, na prática diária da enfermagem, no tratamento e na prevenção dessas lesões. É função do profissional enfermeiro avaliar a lesão, prescrever a cobertura indicada para cada tipo de ferida, além de orientar e supervisionar a equipe de enfermagem na execução do curativo(1). O tratamento de pacientes com lesões de pele, no âmbito hospitalar, necessita do envolvimento de uma equipe multiprofissional. Tais lesões são responsáveis por longas permanências hospitalares, na maioria das vezes em decorrência de tratamentos prolongados, sobretudo em pacientes com a mobilidade comprometida ${ }^{(2)}$. As feridas são consideradas um problema grave, responsáveis por significativos índices de morbimortalidade ${ }^{(3)}$.

Ao identificar os tipos de lesões cutâneas mais frequentes que acometem os pacientes hospitalizados, o enfermeiro necessita planejar, organizar, implementar e avaliar as ações do cuidado prestado às pessoas com lesões agudas e crônicas, objetivando minimizar o tempo de internação e a consequente diminuição dos custos por internações prolongadas ${ }^{(4)}$.

O conceito de lesão de pele ou ferida é tão variado quanto os tipos existentes, sendo definido como a ruptura estrutural e fisiológica do tegumento cutâneo, da membrana mucosa ou de qualquer parte do corpo, podendo ser causada por agentes físicos, químicos ou biológicos. As feridas variam em extensão e profundidade, podendo ser superficiais, quando limitadas à epiderme, à derme e à hipoderme, ou profundas, quando fáscias, músculos, aponeuroses, articulações, cartilagens, tendões, ligamentos, ossos, vasos e órgãos cavitários são atingidos ${ }^{(5)}$. A ferida é aguda quando há ruptura da vascularização com desencadeamento imediato do processo de hemostasia e crônica quando há desvio na sequência do processo cicatricial fisiológico, sendo caracterizada por uma resposta mais proliferativa do que exsudativa ${ }^{(4)}$.

Entre as feridas de maior incidência no ambiente hospitalar estão as lesões por pressão (LP), que frequentemente são complicações decorrentes de um quadro clinico instável e acarretam na ampliação da internação do paciente ${ }^{(6)}$. Assim, faz-se necessária a aplicação de escalas para avaliar o risco e prevenir esse tipo de lesão. São vários os tipos de escalas, dentre as quais a Escala de Braden (EB) é uma das mais utilizadas e consiste em seis subitens, que são: percepção sensorial - capacidade de responder à pressão e ao desconforto; umidade - nível em que a pele é exposta; atividade - grau de atividade física; mobilidade - disposição de controlar a posição do corpo; nutrição - padrão de consumo alimentar; fric- ção - quando ocorre atrito entre superfícies; cisalhamento - quando o paciente desliza na cama, o esqueleto e os tecidos se movimentam, mas a pele permanece inerte ${ }^{(7)}$. Nesse contexto, para identificar a complexidade assistencial, pode-se utilizar a escala Sistema de Classificação de Pacientes $(\mathrm{SCP})^{(8)}$. A escala SCP consiste em um instrumento para estimar as necessidades diárias dos pacientes em relação à assistência de enfermagem a partir de 1960(8).

A partir do exposto, o objetivo geral deste estudo foi caracterizar os pacientes com lesões de pele hospitalizados nas Unidades de Internação Clínico-Cirúrgica de um hospital privado de Porto Alegre/RS.

\section{MÉTODO}

\section{Tipo de estudo}

Trata-se de um estudo transversal.

\section{Participantes da pesquisa (população e amostra, crité- rios de inclusão e exclusão);}

Os participantes foram 45 pacientes com lesões de pele hospitalizados na internação clínica/cirúrgica no ano de 2015. Como critério de inclusão, o paciente deveria ter sido avaliado pelo enfermeiro com indicação de curativos especiais, e o registro do procedimento deveria constar no prontuário eletrônico TASY no item "evolução feridas e curativos". Os critérios de exclusão foram: o uso de coberturas especiais para a prevenção de lesões de pele; menos de dois registros de avaliação de lesão de pele, realizada pelo enfermeiro durante o periodo de hospitalização.

\section{Local do estudo}

Hospital privado de média complexidade localizado em Porto Alegre/RS, com capacidade de 151 leitos. O estudo foi realizado nas três unidades de internação clínica/cirúrgica (4으, 5 o e 6 ㅇ andar) constituída por 51 leitos, que atende pacientes clínicos, crônicos, acamados e/ou dependentes, com nível de complexidade assistencial intermediário e semi-intensivo. No 40 andar estão concentrados pacientes da maternidade, com cuidados mínimos.

A equipe de enfermagem nas três unidades é composta por oito enfermeiras, 40 técnicos de enfermagem, duas secretárias e duas auxiliares geral. Na instituição está implantada a Sistematização de Assistência de Enfermagem (SAE) e as enfermeiras desta unidade desenvolvem quatro das cinco fases do Processo de Enfermagem: Avaliação, Diagnóstico, Planejamento e Implementação.

Nas unidades de internação os pacientes são avaliados diariamente pelo enfermeiro pelo Sistema de Classificação de Pacientes $^{(8)}(\mathrm{SCP})$. Na admissão é realizada avaliação do risco 
de lesão por pressão com a Escala de Braden ${ }^{(7)}$, que é repetida semanalmente.

\section{Coleta dos dados}

A coleta de dados foi realizada no prontuário eletrônico (item: "evolução feridas e curativos"), desenvolvida na unidade de internação clínica/cirúrgica.

\section{Procedimentos de análise}

Os dados foram inseridos e analisados no programa estatístico Statistical Package for Social Sciences (SPSS) versão 21.0 e analisados por estatística descritiva. Nas análises de estatística inferencial, foram realizados o teste t de Student e a análise de variância para comparações múltiplas (ANOVA) com post hoc de Tukey. Foi considerado significativo um $p<0,05$.

\section{Procedimentos éticos}

As questões éticas seguiram as recomendações da Resolução $n^{\circ}$ 466/12 do Conselho Nacional de Saúde do Ministério da Saúde ${ }^{(9)}$. O projeto de pesquisa foi aprovado pelo Comitê de Ética em Pesquisa da Universidade do Vale do Rio dos Sinos ( $n$ ㅇ 1.786.403 e CAAE 60785916.4.0000.5344).

\section{RESULTADOS}

A pesquisa foi realizada com análise dos registros dos prontuários de 45 pacientes com lesão de pele hospitalizados, que atenderam os critérios de inclusão. Dos quais $55,3 \%$ (25) eram mulheres, média de idade foi 60,7 \pm 18,9 e 48,9\% (22) haviam cursado até o ensino fundamental. Na Tabela 1 apresenta-se a distribuição quanto ao motivo de internação, às comorbidades associadas, à mobilidade, à continência/eliminações, ao tempo de hospitalização e ao desfecho

Tabela 1 - Distribuição dos pacientes com lesão de pele internados nas Unidades de Internação Clínico-Cirúrgica quanto ao motivo de internação, às comorbidades associadas, à continência/eliminações, à mobilidade, ao tempo de hospitalização e ao desfecho. Porto Alegre,2017.

\begin{tabular}{lc}
\hline Variáveis & $\mathbf{N}=\mathbf{4 5}$ \\
\hline Motivo de internação & $15(33,3)$ \\
Vascular & $10(22,2)$ \\
Ferida Infectada & $8(17,7)$ \\
Clínico & $6(13,3)$ \\
Cirúrgico & $6(13,3)$ \\
Desbridamento de ferida & \\
\hline Comorbidades associadas & $25(55,5)$ \\
HAS & $9(20,0)$ \\
DM II & \\
DM I & $5(11,1)$ \\
Vaculopatia periférica & $3(6,7)$ \\
Outros & $3(6,7)$
\end{tabular}

\begin{tabular}{|lc|}
\hline Mobilidade & $21(45,7)$ \\
\hline Acamado & $14(30,4)$ \\
\hline Deambula normalmente & $10(23,9)$ \\
\hline Deambula com auxilio & $18(69,2)$ \\
\hline Continência/Eliminações & $21,0(12,0-59,7)$ \\
\hline Uso de Fraldas & \\
\hline Tempo de hospitalização (dias) & $40(87,0)$ \\
\hline Desfecho & $5(13,0)$ \\
\hline Alta & Óbito \\
\hline
\end{tabular}

Legenda: *Variáveis dicotômicas apresentadas por N (\%); \#Variáveis contínuas apresentadas por média (desvio padrão) ou mediana (amplitude); DM: diabetes Mellitus; HAS: hipertensão arterial sistêmica.

Nota: dados descritos em mediana (intervalo interquartil).

Quanto às regiões do corpo acometidas pelas lesões de pele, membros inferiores (30,3\%), regiões lombo-sacra $(28,3 \%)$ e trocantérica (17,3\%). Na Tabela 2, há a caracterização das lesões de pele quanto ao tipo de ferida, bem como quanto ao aspecto da borda, da pele adjacente e do exsudato, à realização do desbridamento cirúrgico, e ao tipo de coberturas utilizadas para os curativos. A Tabela 3 apresenta a distribuição dos pacientes com lesão de pele quanto à pontuação da Escala de Braden e do SCP

Tabela 2 - Distribuição das lesões de pele dos pacientes internados nas Unidades de Internação Clínico-Cirúrgica quanto ao tipo da lesão, ao aspecto da borda, da pele adjacente e do exsudato, e ao desbridamento. Porto Alegre,2017.

\begin{tabular}{|lc|}
\hline Variáveis & $\mathbf{N}=\mathbf{4 5}$ \\
\hline Tipo de lesão & \\
Lesão por pressão & $20(44,4)$ \\
FO complicada & $14(31,1)$ \\
Úlcera varicosa & $5(11,1)$ \\
Erisipela & $1(2,2)$ \\
Pé diabético & $3(6,6)$ \\
Outros & $2(4,4)$ \\
\hline Aspecto da borda*1 & \\
\hline Irregular & $16(84,2)$ \\
Regular & $3(15,8)$ \\
\hline Pele adjacente ${ }^{* 1}$ & \\
\hline Íntegra & $12(52,2)$ \\
Macerada & $7(30,4)$ \\
Dermatite periferida & $4(17,4)$ \\
\hline Aspecto exsudato*1 & $12(38,7)$ \\
\hline Seroso & $8(25,8)$ \\
Purulento & $7(22,5)$ \\
Sanguinolento & \\
Serosanguinolento & $21(75,0)$ \\
\hline Desbridamento & \\
\hline Sim & $(12,9)$ \\
\hline
\end{tabular}


Legenda: *Variáveis dicotômicas apresentadas por N (\%); \#Variáveis contínuas apresentadas por média (desvio padrão) ou mediana (amplitude). ${ }^{1}$ Para definir o percentual, foram considerados os registros das evoluções nas quais constam informações sobre o aspecto da borda da lesão (19), da pele adjacente à lesão (23) e do exsudato (31).

$\mathrm{Na}$ instituição em estudo, a EB é feita na admissão do paciente, e repetida semanalmente e a cada alteração do seu estado geral. Já a SCP é feita na admissão do paciente e repetida diariamente. Os dados analisados são apresentados na Tabela 5.

Tabela 3 - Distribuição dos pacientes com lesões de pele internados nas Unidades de Internação Clínico-Cirúrgica quanto à idade, ao tempo de hospitalização e à avaliação com a EB e com o SCP. Porto Alegre,2017.

\begin{tabular}{|lll|}
\hline \multicolumn{1}{|c}{ Variáveis } & $\mathbf{5 0}$ andar & 69 andar \\
\hline Idade (anos) & $74,5 \pm 16,4^{*}$ & $64,6 \pm 14,4$ \\
\hline Tempo de hospitalização & $28,6 \pm 14,0$ & $56,3 \pm 12,3$ \\
\hline Braden inicial & $14,9 \pm 3,8$ & $14,5 \pm 6,4$ \\
\hline Braden final & $10,3 \pm 6,5$ & $11,6 \pm 4,8$ \\
\hline SCP inicial & $32,3 \pm 5,9^{*}$ & $29,3 \pm 6,7$ \\
\hline SCP final & $30,8 \pm 8,7^{*}$ & $28,8 \pm 6,0$ \\
\hline
\end{tabular}

Legenda: *Apresentam as diferenças significativas.

Nota: Diferença das médias avaliadas por ANOVA e post hoc de Tukey.

Quanto às coberturas, o número ultrapassou 100\% devido ao uso de mais de uma cobertura em pacientes com mais de uma lesão ou o curativo com associação de coberturas. As mais frequentes foram: ácidos graxos essenciais 72\% (41); alginato de cálcio 31,6\% (18); hidrofibra de prata 24,6\% (14); gaze não aderente 22,8\% (13) e; papaína 10\% 19,3\% (11).

\section{DISCUSSÃO}

Quanto ao sexo, à idade e à escolaridade, identificou-se que a maioria era feminino (54,3\%), idosa $(67,0 \pm 18,9)$ e $48,9 \%$ cursou até o ensino fundamental. Maior incidência de lesões em pacientes do sexo feminino pode também ser evidenciados na literatura, em que se alega que as mulheres apresentam três vezes mais chances de desenvolver lesões de pele (10). As mulheres têm maior longevidade que os homens, o que as leva a períodos mais longos de doenças crônicas com o aumento do tempo médio de institucionalização ${ }^{(11)}$. Isso as deixa mais suscetiveis ao desenvolvimento de lesões de pele. Ademais, o idoso também está mais suscetivel ao agravo devido às alterações fisiológicas, as quais acometem a função e o aspecto da pele ${ }^{(12)}$. Também ocorre diminuição da resposta inflamatória, da sintese de colágeno e conjuntamente, há um aumento da fragilidade capilar e do tempo de epitelização, resultando em retardamento das fases de cicatrização ${ }^{(13)}$

O nivel educacional pode interferir no desenvolvimento da capacidade de compreensão do tratamento indicado e na prática do autocuidado ${ }^{(14,15)}$. Nesta perspectiva, é necessário que o enfermeiro desenvolva, junto com a equipe assistencial, um processo de ensino-aprendizagem que considere o perfil de escolaridade. É importante que os pacientes aprendam a controlar os fatores que podem interferir no processo de cicatrização das lesões de pele(16)

Os problemas vasculares (33,3\%) foi o motivo de internação mais recorrente. As comorbidades associadas à HAS e ao DM foram as mais frequentes, representando 55,5\% e $31,1 \%$ dos casos, respectivamente. Comorbidades são consideradas um fator de causa intrínseca no desenvolvimento de lesões de pele, sobretudo nas doenças crônicas como as cardiovasculares e o DM ${ }^{(17)}$.

Tanto a HAS como o DM interferem e comprometem o processo de cicatrização devido às complicações vasculares (15). Além disso, associam-se às dislipidemias, afetando os leitos vasculares e a microcirculação, causando disfunção endotelial e aterosclerose, com aumento do risco de desenvolvimento de úlceras e lesões nos membros inferiores ${ }^{(18)}$

Quanto à mobilidade dos pacientes deste estudo, 45,7\% estavam acamados, o que contribuiu para o aumento dos riscos de se ter lesões de pele. Pacientes hospitalizados, podem apresentar diferentes graus de comprometimento motor, frequentemente permanecendo limitados ao leito, acamados por longos períodos, e desenvolvendo algum tipo de lesão de pele ${ }^{(2)}$. Limitações da mobilidade causadas por outras sequelas também são fatores associados ao risco de desenvolver uma lesão de pele ${ }^{(19)}$.

Quanto à continência/eliminações, a maioria dos pacientes fazia uso de fraldas, o que expõe a pele à umidade, ao microclima e a possiveis lesões cutâneas. O uso de fralda determina higiene perineal e de hidratação local da pele após as eliminações, pois o contato da pele com a urina por longos periodos ocasiona a maceração do tecido ${ }^{(20)}$

O tempo médio de internação hospitalar foi de 21,0 dias, variando entre 12,0 e 59,7 dias. No total, 87\% tiveram alta para casa. Em relação às lesões de pele, este tipo de agravo, prolonga o tempo de hospitalização devido aos tratamentos serem prolongados ${ }^{(2)}$.

As LP foram as mais frequentes, representando 44,4\% das internações. Outro estudo realizado aponta esse tipo de 
agravo como uma das principais complicações que podem acometer os pacientes hospitalizados ${ }^{(21)}$. Resultados convergentes com pesquisas realizadas em Unidades de Internação Clínico-Cirúrgica indicam que os pacientes internados nestas unidades têm maiores chances de desenvolver LP durante a hospitalização ${ }^{(12,22)}$

A LP é considerada um problema para os serviços de saúde e envolve diretamente a equipe interdisciplinar ${ }^{(2)}$. Trata-se de um fenômeno que, quando associado às condições clínicas do paciente, pode exceder os cuidados de enfermagem, pois sua etiologia é multifatorial, incluindo fatores intrínsecos e extrínsecos, como idade, comorbidades, entre outros ${ }^{(23)}$. Entretanto, os profissionais de enfermagem desempenham papel crucial na prevenção da LP na medida em que asseguram a mobilidade, a oferta e administração de alimentos e a menor exposição à umidade, além de contribuírem para evitar a fricção e o cisalhamento ${ }^{(24)}$.

As LP ocasionadas durante a internação aumentam os dias de internação e o custo do tratamento e reduzem a qualidade de vida dos pacientes ${ }^{(19)}$. Nesse contexto, são necessárias ações de cuidado com medidas preventivas de LP e com a adoção de protocolos que possibilitem a sistematização do cuidado(7).

A borda irregular representou $84,2 \%$. Em 75\% foi realizado desbridamento cirúrgico pelo médico e pelo enfermeiro habilitado. A realização do desbridamento cirúrgico por enfermeiro tecnicamente apto e legalmente assistido vem somar efetivamente esforços à equipe de saúde, garantindo uma assistência de qualidade ${ }^{(25)}$.

Neste estudo, os curativos ou coberturas especiais mais utilizadas, foram: ácidos graxos essenciais (AGE) (26); alginato de cálcio(27); hidrofibra de prata ${ }^{(28)}$; gaze não aderente ${ }^{(29)}$; e papaina $10 \%{ }^{(30)}$.

A cobertura ideal deve: promover impermeabilidade à água e aos fluídos; manter meio úmido e a temperatura adequada; proteger a ferida contra possiveis traumas mecânicos e infecções; viabilizar as trocas gasosas, absorver o exsudato e proporcionar o desbridamento, aliviando a dor (26). Portanto, a escolha do curativo deve ser realizada com a avaliação criteriosa da ferida, considerando a fisiologia da cicatrização e os elementos que possam contribuir para acelerar esse processo(31)

Por fim, o planejamento da assistência de enfermagem a pacientes internados tem sido cada vez mais orientado pela utilização de escalas e diretrizes que identifiquem as condições dos pacientes ${ }^{(32)}$. Neste estudo, quanto aos escores do SCP com as classificações de risco da EB, constatou-se que as duas escalas estão associadas entre si, confirmando que os pacientes internados nas Unidades de Internação Clíni-
co-Cirúrgica do $5^{\circ}$ e $6^{\circ}$ andar com classificação de cuidados intermediários no SCP concentram-se na classificação de risco Moderado/Elevado para o desenvolvimento de lesão por pressão (LP), o que justifica o predomínio da LP como a lesão mais frequente entre as lesões de pele. Como fator limitante, o $4^{\circ}$ andar foi desconsiderado da análise comparativa, pois se trata de uma unidade distinta das demais, por concentrar pacientes da maternidade, com cuidados mínimos.

A EB tem como objetivo identificar o risco do paciente em desenvolver LP(19). A SCP, por sua vez, é utilizada para determinar o grau de dependência do paciente em relação à equipe de enfermagem, à alocação de recursos humanos, à monitorização da produtividade e aos custos da assistência de enfermagem ${ }^{(33)}$. Sendo assim, ambas as escalas podem contribuir para a identificação de possiveis riscos.

A avaliação e monitorização das feridas permitem identificar o estado inicial da lesão e o sucesso do tratamento indicado, tornando premente a necessidade de registros confiáveis e universais ${ }^{(34)}$. Portanto, a documentação e os registros de enfermagem são ferramentas importantes para qualificar o processo do cuidado e a SAE, promovendo a eficácia das intervenções.

\section{Limitações do estudo}

As limitações deste estudo estão relacionadas aos registros nos prontuários que poderiam ser mais completos de modo a favorecer o estabelecimento de juízos de avaliação.

\section{Contribuições para a prática}

O estudo apresenta subsídios para implantação de ambulatórios para o acompanhamento de feridas complexas.

\section{CONCLUSÃO}

Considera-se que, a caracterização do paciente hospitalizado com lesões de pele, contribui para planejar e implantar assistência integral e sistematizada. Além disso, pode subsidiar atualização de protocolos clínicos específicos de prevenção e tratamento das lesões de pele, em especial a LP. Também se espera que se possa priorizar capacitações das equipes de enfermagem para a sistematização do cuidado, preconizando a prevenção das lesões de pele, com o objetivo de se construir uma instituição reconhecida na excelência por ter foco na prevenção e no tratamento das feridas com coberturas especiais.

CONTRIBUIÇÃO DOS AUTORES: Concepção e/ou desenho, análise e interpretação dos dados, redação do artigo, revisão crítica, revisão final: Rochelli Bernardes Stefanello; Silvana Mara Janing Prazeres, Fernanda Silva dos Santos, Joel Rolim Mancia; Sandra Maria Cezar Leal. 


\section{REFERÊNCIAS}

1 Rocha ACAA, Carneiro FAS, Souza MS. Tratamento domiciliar de feridas crônicas: relato de experiência da extensão na prática do cuidar. Rev Ciência e Estudos Acadêmicos de Medicina. [Internet] 2014 [cited 2019 May 5] ; (2):20-30. Disponivel em: <http:// periodicos.unemat.br/index.php/revistamedicina/article/view/354/338>

2 Mattos RM, Melo FBS, Araújo AKC, Gomes GMS, Vasconcelos LDS, LDT Souza. Educação em saúde aos trabalhadores de enfermagem e acompanhantes sobre prevenção e tratamento de lesões de pele em dois hospitais de Petrolina- PE. Interfaces - Revista de Extensão. [Internet] 2015 [cited 2019 May 5] 3(1):22-32. Disponivel em: <https://www.ufmg.br/proex/revistainterfaces/index.php/ IREXT/article/view/44>

3 Oliveira FP, Oliveira BGRB, Santana RF, Paula BS, Candido JSC. Classificações de intervenções e resultados de enfermagem em pacientes com feridas: mapeamento cruzado. Rev Gaúcha Enferm. [Internet] 2016 [cited 2019 May 25]; 37(2) :e55033.

. Disponivel em: http://www.scielo.br/pdf/rgenf/v37n2/en_0102-6933-rgenf-1983-144720160255033.pdf

4 Chibante CLP. Santos FHE, Santos TD. Perfil de clientes hospitalizados com lesões cutâneas. Perfil de clientes hospitalizados com lesões cutâneas. Rev Cubana Enferm. [Internet] 2015 [cited 2019 Jun 15]; 31(4). Disponivel em: <http://www.revenfermeria.sld.cu/ index.php/enf/article/view/895/136>

5 Geovanini T. Classificação e tipos de feridas. In: Geovanini T. Tratado de feridas e curativos: enfoque multiprofissional. São Paulo: Rideel; 2014

6 Ferreira CJM, Chacon JMF, Blanes L, Góis AFT, Ferreira LM, Zucchi P. Aspectos epidemiológicos do paciente com úlcera por pressão na Unidade de Terapia Intensiva do pronto-socorro de um hospital de ensino de São Paulo. Saúde Coletiva. [Internet] 2013 [cited 2019 May 5]; 10(59):14-19. Disponivel em: <http://www.redalyc.org/pdf/842/84228211003.pdf>

7 Otto C, Schumacher B, Wiese LPL, Ferro C, Rodrigues RA. Fatores de risco para o desenvolvimento de lesão por pressão em pacientes críticos. Enferm foco. [Internet] 2019 [cited 2019 May 5]; 10(1): 07-11. Disponivel em: http://biblioteca.cofen.gov.br/wpcontent/uploads/2019/03/Fatores-De-Risco-Para-o-Desenvolvimento-De-Les\%C3\%A3o-Por-Press\%C3\%A3o-Em-PacientesCr\%C3\%ADticos.pdf

8 Fugulin FMT, Gaidzinski RR, Kurcgant P. Sistema de Classificação de Pacientes: identificação do perfil assistencial dos pacientes das unidades de internação do HU-USP. Rev Latino-am Enferm. [Internet] 2005 [cited 2019 May 5]; 13(1):72-78. Disponivel em: <http:// www.scielo.br/pdf/rlae/vl3nl/vl3nlal2.pdf>

9 Conselho Nacional de Saúde (BR)Resolução n 466/12. Pesquisa em seres humanos. Homologada em 12 dezembro de 2012. Diário Oficial da União Brasilia 2013 jun. 13; (12):Seção 1 p.59. Disponivel em: <http://conselho.saude.gov.br/resolucoes/2012/Reso466.pdf>.

10Oliveira BGRB, Nogueira GA, Carvalho MR, Abreu AM. Caracterização dos pacientes com úlcera venosa acompanhados no Ambulatório de Reparo de Feridas. Rev Eletrônica Enferm. [Internet] 2012 [cited 2019 Ago 12]; 14(1):156-163. Disponivel em: <https:// www.revistas.ufg.br/fen/article/view/10322>.

11 AF Lucena, CT Santos, Silva PAG, Almeida MA, Dias VLM, Friedrich MA. Perfil clínico e diagnósticos de enfermagem de pacientes em risco para úlcera por pressão. Rev Latino-am Enferm. [Internet] 2011 [cited 2019 Ago 12]; 19(3):523-530. Disponivel em: <http://www. scielo.br/scielo.php?pid=S0104-11692011000300011Escript=sci_arttextEtlng=pt>.

12 Barreto APCP, Araújo BC, Vieira JCM. Perfil sociodemográfico e clínico de pacientes com úlceras por pressão. Rev Enferm UFPE. [Internet] 2011[cited 2019 Oct 5]; 5(9):215-2-60. Disponivel em: <http://www.revista.ufpe.br/revistaenfermagem/index.php/revista/ article/viewFile/1838/pdf_681>.

13 Costa A, Dieamant G, Pereira ESP, Fávaro R, Assumpção EC, Ota FS et al. Eficácia in vitro e clínica do uso cosmético tópico de fitoestrógenos em pelefotoenvelhecida. Surgicaland Cosmetic Dermatology. [Internet] 2012 [cited 2019Ago 15]; 4(2):137-149. Disponivel em: $\quad$ <http://webcache.googleusercontent.com/search?q=cache:1DzMp_9yEbAJ:www.surgicalcosmetic.org.br/exportar-pdf/4/4_ n2_200_pt/Eficacia-in-vitro-e-clinica-do-uso-cosmetico-topico-de-fitoestrogenos-em-pele-fotoenvelhecida+\&cd=18hl=ptBREct=clnkEgl=br>.

14 Pérez Rodriguez MC, Godoy S, Mazzo A, Nogueira PC, Trevizan MA, Mendes IAC. Cuidado com os pés diabéticos antes e após intervenção educativa. Rev Electrônica Trim Enferm. [Internet] 2013 [cited 2019 Ago 12]; (29):53-62. Disponivel em: <http://scielo.isciii. es/pdf/eg/v12n29/pt_clinica3.pdf>.

15 Simão CMF, Caliri MHL, Santos CB. Concordância entre enfermeiros quanto ao risco dos pacientes para úlcera por pressão. Acta Paul Enferm. [Internet] 2013 [cited 2019 May 5]; 26(1). Disponivel em: <http://www.scielo.br/scielo.php?script=sci_ arttextEpid=S0103-21002013000100006>

16 Camacho ACLF, Santos RC, Joaquim FL, Louredo DS, Morais IM, Silva EA. Estudo comparativo sobre a capacidade funcional 
de pacientes adultos e idosos com úlceras venosas. Rev Pesquisa: Cuidado é Fundamental. [Internet] 2015 [cited 2019 April 5]; 7(1):1954-1966. Disponivel em: <http://www.seer.unirio.br/index.php/cuidadofundamental/article/viewFile/3505/pdf_1435>

17 Silva PD, Barbosa MH, Araújo DF, Oliveira LP, Melo AF. Úlcera por pressão: avaliação de fatores de risco em pacientes internados em um hospital universitário. Rev Eletrônica Enferm. [Internet] 2011 [cited 2019 Jul 5] ; 13(1):118-123. Disponivel em: <https://www. fen.ufg.br/fen_revista/v13/nl/pdf/v13nlal3.pdf>.

18 Geovanini T. Fatores de risco para o desenvolvimento de úlceras e lesões da pele. In: Geovanini T. Tratado de feridas e curativos: enfoque multiprofissional. São Paulo: Rideel; 2014

19 Menegon DB, Bercini RR, Santos CT, Lucena AF, Pereira AGS, Scain SF. Análise das subescalas de Braden como indicativos de risco para úlcera de pressão. Texto \& Contexto Enferm. [Internet] 2012 [cited 2019 May 5]; 21(4). Disponivel em: <http://www.scielo. br/scielo.php?script=sci_arttext\&pid=S0104-07072012000400016>.

20 Chimentão DMN, Domansky RC. Dermatite associada a incontinência. In: Domansky RC, Borges EL. Manual para prevenção de lesões de pele: recomendações baseadas em evidências. Rio de Janeiro: Rubio; 2012.

21 Chavaglia SRR, Ohl RIB, Ferreira LA, Santiago VR, Abdanur AF, Soares AS. Caracterização de pacientes com lesão cutânea em unidades de internação médica e cirúrgica. Rev Enferm UFPE. [Internet] 2015 [cited 2019 Ago 15]; 9(1):183-192. Disponivel em: https://periodicos.ufpe.br/revistas/revistaenfermagem/article/view/10324

22 Santos CT, Oliveira CM, Pereira AGSP, Suzuki LM, Lucena AF. Indicador de qualidade assistencial úlcera por pressão: análise de prontuário e de notificação de incidente. Rev Gaúcha Enferm. [Internet] 2013 [cited 2019 Oct 12]; 34(1). Disponivel em: <http://www. scielo.br/scielo.php?script=sci_arttextEpid=S1983-14472013000100014>.

23 Rogenski NMB, Kurcgant P. Incidência de úlceras por pressão após implementação de um protocolo de prevenção. Rev Latinoam Enferm. [Internet] 2012 [cited 2019 Oct 5]; 20(2):07 telas. Disponivel em: <http://www.scielo.br/pdf/rlae/v20n2/pt_16>.

24 Bavaresco T, Medeiros RH, Lucena AF. Implantação da Escala de Braden em uma unidade de terapia intensiva de um hospital universitário. Rev Gaúcha Enferm. [Internet] 2011 [cited 2019 Oct 5] ; 32(4):703-710. Disponivel em: <http://www.scielo.br/scielo. php?script=sci_arttextEpid=S1983-14472011000400010>

25 Santos ICRV, Oliveira RC, Silva MA. Desbridamento cirúrgico e a competência legal do enfermeiro. Texto \& Contexto Enfermagem. [Internet] 2013 [cited 2019 Oct 5]; 22(1):184-192. Disponivel em: <http://www.scielo.br/pdf/tce/v22nl/pt_22.pdf>

26 Sood A, Granick MS, Tomaselli NL. Wound dressings and comparative effectiveness data. Advances in Wound Care. [Internet] 2014 [cited 2019 Oct 5]; 3(8):511-529. Disponivel em: <https://www.ncbi.nlm.nih.gov/pmc/articles/PMC4121107/>.

27 Fan K, Tang J, Escandon J, Kirsner RS. State of the art in topical wound-healing products. Plastic and Reconstructive Surgery. [Internet] 2011 [cited 2019 May 5]; 127(1 S):44S-59S. Disponivel em: <https://www.researchgate.net/profile/Kenneth_Fan/ publication/49723084_State_of_the_Art_in_Topical_Wound-Healing_Products/links/Odeec515c305abb441000000.pdf>

28 Prazeres SJ, Silva ACB. Técnica e tecnologias no tratamento de feridas. In: Prazeres SJ. Tratamento de feridas: teoria e prática. Porto Alegre: Moriá; 2009.

29 Sasseron MGM. Atualidades em curativos oclusivos e semioclusivos. In: Malagutti W, Kakihara CT. Curativos, estomias e dermatologia: uma abordagem multiprofissional. 3. ed. São Paulo: Martinari; 2015.

30 Guimarães MCSS, Geovanini T. Coberturas e novas tecnologias para o cuidado de feridas. In: Geovanini T. Tratado de feridas e curativos: enfoque multiprofissional. São Paulo: Rideel; 2014.

31 Melo EM, Fernandes VS. Avaliação do conhecimento do enfermeiro acerca das coberturas de última geração. Revista Estima. [Internet] 2011 [cited 2019 May 5]; 9(4):Itela. Disponivel em:

<http://www.revistaestima.com.br/index.php/estima/article/view/69>

32 Urbanetto JS Santos MACN, Poltozi AF, Pechansky ALC, Hax G, Custódio A. A relação entre a dependência de cuidados, risco e úlcera por pressão. Enferm Foco. [Internet] 2012 [cited 2019 Oct 5]; 3(4). Disponivel em: http://revista.cofen.gov.br/index.php/ enfermagem/article/view/384

33 Gaidzinski RR. O dimensionamento do pessoal de enfermagem segundo a percepção de enfermeiras que vivenciam esta prática [tese]. São Paulo: Universidade de São Paulo; 1994. Disponivel em: <http://www.scielo.br/scielo.php?script=sci_ nlinks\&ref=000125\&pid=S0104-1169200500010001200004\&Ing=en>.

34 Antunes J, Carvalho P, Freire T, Marques F. Nursing records and wound healing evolution. Journal of Aging \& Inovation. [Internet] 2015 [cited 2019 May 5]; 4(2):3-10. Disponivel em: <http://journalofagingandinnovation.org/wp-content/uploads/l-registos-feridas.pdf>. 